\title{
A note on the $\gamma$-coefficients of the tree Eulerian polynomial
}

\author{
Rafael S. González D’León* \\ Department of Mathematics \\ University of Kentucky \\ Lexington, KY, U.S.A. 40506-0027 \\ rafaeldleon@uky.edu
}

Submitted: Jun 26, 2015; Accepted: Jan 20, 2016; Published: Feb 5, 2016

Mathematics Subject Classifications: 05A15, 05E05

\begin{abstract}
We consider the generating polynomial of the number of rooted trees on the set $\{1,2, \ldots, n\}$ counted by the number of descending edges (a parent with a greater label than a child). This polynomial is an extension of the descent generating polynomial of the set of permutations of a totally ordered $n$-set, known as the Eulerian polynomial. We show how this extension shares some of the properties of the classical one. A classical product formula shows that this polynomial factors completely over the integers. From this product formula it can be concluded that this polynomial has positive coefficients in the $\gamma$-basis and we show that a formula for these coefficients can also be derived. We discuss various combinatorial interpretations of these coefficients in terms of leaf-labeled binary trees and in terms of the Stirling permutations introduced by Gessel and Stanley. These interpretations are derived from previous results of Liu, Dotsenko-Khoroshkin, BershteinDotsenko-Khoroshkin, González D'León-Wachs and González D'León related to the free multibracketed Lie algebra and the poset of weighted partitions.
\end{abstract}

Keywords: Gamma positivity; Eulerian polynomial; Rooted trees

\section{1 introduction}

A labeled rooted tree $T$ on the set $[n]:=\{1,2, \cdots, n\}$ is a tree whose nodes or vertices are the elements of $[n]$ and such that one of its nodes has been distinguished and called the root. For nodes $x$ and $y$ in $T$ we say that $x$ is the child of $y$ or $y$ is the parent of $x$ if $y$ is the first node following $x$ in the unique path from $x$ to the root of $T$ and we

*Supported by NSF Grant DMS 1202755. 
say that $y=\mathrm{p}(x)$. Nodes that have children are said to be internal otherwise we call a node without children a leaf. If $y$ is the parent of $x$, we say that the edge $\{x, y\}$ of $T$ is descending (and we call $x$ a descent of $T$ ) if the label of $y$ is greater than the label of $x$. We denote $\operatorname{des}(T)$ the number of descents in $T$. Figure 1 shows all the rooted trees on [3] grouped by the number of descents. We draw the trees with the convention that parents come higher than their children and the root is the highest node. We denote $\mathcal{T}_{n}$ the set of rooted trees on $[n]$ and $\mathcal{T}_{n, i}$ the set of trees in $\mathcal{T}_{n}$ with exactly $i$ descents.

For a given $n \geqslant 1$ define

$$
T_{n}(t):=\sum_{T \in \mathcal{T}_{n}} t^{\operatorname{des}(T)}=\sum_{i=0}^{n-1}\left|\mathcal{T}_{n, i}\right| t^{i},
$$

the descent generating polynomial of $\mathcal{T}_{n}$. We call $T_{n}(t)$ the tree Eulerian polynomial in analogy with the classical polynomial $A_{n}(t)=\sum_{\sigma \in \mathfrak{S}_{n}} t^{\operatorname{des}(\sigma)}$, that is the descent generating polynomial of the set $\mathfrak{S}_{n}$ of permutations of $[n]$. We can identify the permutations in $\mathfrak{S}_{n}$ with the set of rooted trees on $[n]$ that have $n-1$ internal nodes, each of them having a unique child (and so containing a unique leaf). It is not hard to see that our definition of a descent on this set of trees coincides with the classical definition of descent in a permutation so the polynomial $T_{n}(t)$ is an extension of the polynomial $A_{n}(t)$. The polynomials $A_{n}(t)$ have been extensively studied in the literature and are known with the name of Eulerian polynomials since Euler was one of the first in studying them (see [20]). The Eulerian polynomial $A_{n}(t)=\sum_{k=0}^{n-1} A_{n, i} t^{i}$ have degree $n-1$ and its coefficients satisfy the relation

$$
A_{n, i}=A_{n, n-1-i} .
$$

For example, the Eulerian polynomial for $n=3$ is $A_{3}(t)=1+4 t+t^{2}$. A polynomial that satisfies Equation 1.2 is called symmetric or palindromic.

It is a simple observation that a symmetric polynomial $f(t)=\sum_{k=0}^{d} f_{i} t^{i}$ of degree $d$ with $f_{i} \in \mathbb{Z}$ can be written in the form

$$
\sum_{i=0}^{d} f_{i} t^{i}=\sum_{i=0}^{\left\lfloor\frac{d}{2}\right\rfloor} \gamma_{i} t^{i}(1+t)^{d-2 i},
$$

where the coefficients $\gamma_{i} \in \mathbb{Z}$, i.e., the set $\left\{t^{i}(1+t)^{d-2 i}\right\}_{i=0}^{\left\lfloor\frac{d}{2}\right\rfloor}$, where $\lfloor\cdot\rfloor$ is the integer floor function, is a basis (known as the $\gamma$-basis) for the space of symmetric polynomials of degree $d$ with integer coefficients. If $\gamma_{i} \geqslant 0$ for all $i$ then we say that the polynomial $f(t)$ is $\gamma$-nonnegative and if $\gamma_{i}>0$ for all $i \leqslant\left\lfloor\frac{d}{2}\right\rfloor$ then we say that the polynomial $f(t)$ is $\gamma$-positive. It is a result of Foata and Schützenberger ([8]) that $A_{n}(t)$ is $\gamma$-positive. And it follows from the work of Foata and Strehl in [9] that its coefficients $\gamma_{i}$ have a nice combinatorial interpretation (see also [18]). Indeed, let $\widehat{\mathfrak{S}}_{n}$ be the set of permutations in $\mathfrak{S}_{n}$ that have no two adjacent descents and no descent in the last position. Then

$$
\gamma_{j}=\left\{\sigma \in \widehat{\mathfrak{S}}_{n} \mid \operatorname{des}(\sigma)=j\right\}
$$


For example, $A_{3}(t)=(1+t)^{2}+2 t$ with $\gamma_{0}=1$ and $\gamma_{1}=2$. The permutations in $\widehat{\mathfrak{S}}_{3}$ are, 123 with no descents and; 213 and 312 with one descent. Gal [10] and Brändén $[2,3]$ have introduced the use of the $\gamma$-basis in different contexts. Gal conjectured that the $\gamma$-coefficients of the $h$-polynomial of a flag simple polytope are all nonnegative. In particular, $A_{n}(t)$ is the $h$-vector of the permutahedron that is a flag simple polytope so Gal's conjecture is confirmed in this case. Postnikov, Reiner and Williams [17] have confirmed Gal's conjecture for the family of chordal nestohedra that is a large family of flag simple polytopes. For more information about $\gamma$-nonnegativity see [4].

We will show that the properties discussed above for the Eulerian polynomial $A_{n}(t)$ are also shared by the polynomial $T_{n}(t)$ in a similar fashion. The degree of $T_{n}(t)$ is also $n-1$ and it is easy to see from the definition of a descent that

$$
\left|\mathcal{T}_{n, i}\right|=\left|\mathcal{T}_{n, n-1-i}\right|
$$

so $T_{n}(t)$ is also symmetric. Indeed there is a natural bijection $\mathcal{T}_{n, i} \simeq \mathcal{T}_{n, n-1-i}$ where the image of a labeled rooted tree $T \in \mathcal{T}_{n, i}$, is the tree in $\mathcal{T}_{n, n-1-i}$ with the same shape of $T$ but where each label $i$ has been replaced by $n+1-i$. For the example in Figure 1, $T_{3}(t)=2+5 t+2 t^{2}$.

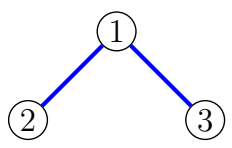

$\int_{3}^{1}$

$\operatorname{des}(T)=0$
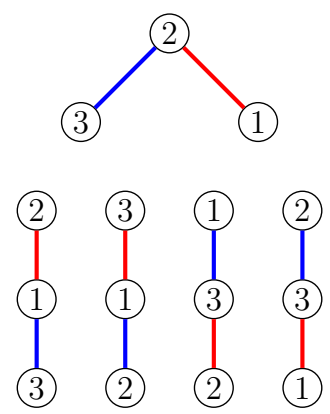

$\operatorname{des}(T)=1$
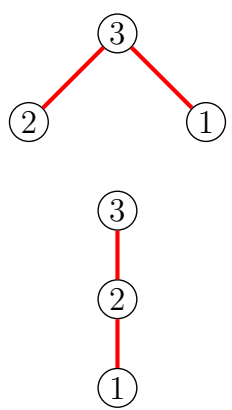

$\operatorname{des}(T)=2$

Figure 1: All labeled rooted trees on [3]

The following nice product formula for $T_{n}(t)$ can be obtained from the results in [7] (see also [12] and [6]).

Theorem 1. For $n \geqslant 1$,

$$
\sum_{i=0}^{n-1}\left|\mathcal{T}_{n, i}\right| t^{i}=\prod_{i=1}^{n-1}((n-i)+i t)
$$

In particular, setting $t=1$ in (1.5) reduces to the classical formula $\left|\mathcal{T}_{n}\right|=n^{n-1}$. Equation (1.5) implies that all the roots of this polynomial are real and negative. It is known and not difficult to show that a real-rooted symmetric polynomial with nonnegative real coefficients is $\gamma$-nonnegative (see $[4,10]$ ). For example $T_{3}(t)=2(1+t)^{2}+t$, so $\gamma_{0}=2$ and $\gamma_{1}=1$. Although $T_{n}(t)$ is not in general an $h$-vector of a convex polytope (for example 
$h_{0} \neq 1$ for $n \geqslant 3$ ), it is of interest to find combinatorial formulas and interpretations of nonnegative $\gamma$-coefficients of general symmetric polynomials.

Let $\gamma_{j}\left(T_{n}(t)\right)$ denote the $j$-th $\gamma$-coefficient of the symmetric polynomial $T_{n}(t)$. Equation (1.5) can be used to find a formula for the coefficients $\gamma_{j}$ of $T_{n}(t)$. The values of $\gamma_{j}\left(T_{n}(t)\right)$ for $n=1, \ldots, 7$ appear in Table 1 .

Theorem 2. For $n \geqslant 1$,

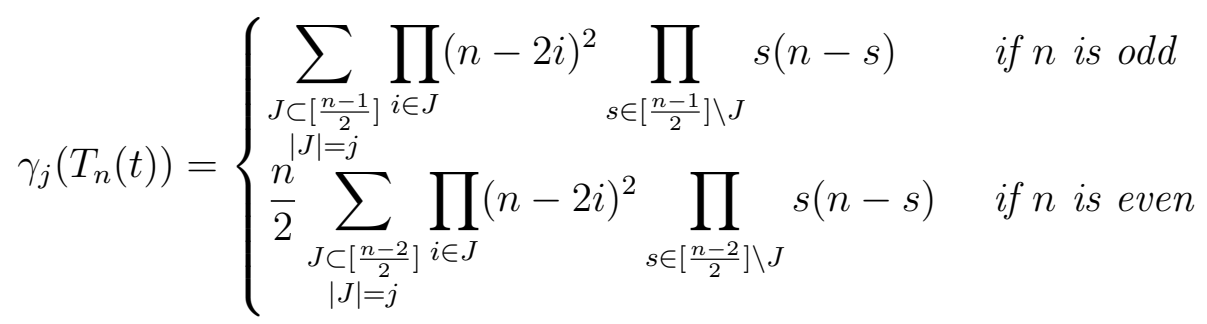

Proof. If we multiply

$$
\begin{aligned}
(n-i+i t)(i+(n-i) t) & =(n-i) i+\left[(n-i)^{2}+i^{2}\right] t+(n-i) i t^{2} \\
& =(n-i) i\left(1+t^{2}\right)+\left[(n-i)^{2}+i^{2}\right] t \\
& =(n-i) i\left(1+2 t+t^{2}\right)+\left[(n-i)^{2}-2 i(n-i)+i^{2}\right] t \\
& =(n-i) i(1+t)^{2}+(n-2 i)^{2} t .
\end{aligned}
$$

Equation 1.5 can be written as

$$
T_{n}(t)= \begin{cases}\prod_{i=1}^{\frac{n-1}{2}}\left[(n-i) i(1+t)^{2}+(n-2 i)^{2} t\right] & \text { if } n \text { is odd } \\ \frac{n}{2}(1+t) \prod_{i=1}^{\frac{n-2}{2}}\left[(n-i) i(1+t)^{2}+(n-2 i)^{2} t\right] & \text { if } n \text { is even }\end{cases}
$$

implying Formula 1.6.

\begin{tabular}{|c|c|c|c|c|}
\hline $\boldsymbol{n} / \boldsymbol{j}$ & $\mathbf{0}$ & $\mathbf{1}$ & $\mathbf{2}$ & $\mathbf{3}$ \\
\hline $\mathbf{1}$ & 1 & & & \\
\hline $\mathbf{2}$ & 1 & & & \\
\hline $\mathbf{3}$ & 2 & 1 & & \\
\hline $\mathbf{4}$ & 6 & 8 & & \\
\hline $\mathbf{5}$ & 24 & 58 & 9 & \\
\hline $\mathbf{6}$ & 120 & 444 & 192 & \\
\hline $\mathbf{7}$ & 720 & 3708 & 3004 & 225 \\
\hline
\end{tabular}

Table 1: Values of $\gamma_{j}\left(T_{n}(t)\right)$ for $n=1, \ldots, 7$. 
The purpose of this note is to present four different combinatorial interpretations for the coefficients $\gamma_{j}$ that are consequences of results in the work of Liu [15], DotsenkoKhoroshkin [5], Bershtein-Dotsenko-Khoroshkin [1], González D'León-Wachs [14] and González D'León [13]. We present now one of these combinatorial interpretations, whose proof will be given in Section 2 .

A planar leaf-labeled binary tree with label set $[n]$ is a rooted tree (a priori without labels) in which the set of children of every internal node is a totally ordered set with exactly two elements (the left and right children) and where each leaf has been assigned a unique element from the set $[n]$. By a subtree in a rooted tree $T$ we mean the rooted tree induced by the descendents of any node $x$ of $T$, including and rooted at $x$. We say that a planar leaf-labeled binary tree with label set $[n]$ is normalized if in each subtree, the leftmost leaf is the one with the smallest label. We denote the set of normalized binary trees with label set $[n]$ by $\mathrm{Nor}_{n}$. All normalized trees with leaf labels in [3] are illustrated in Figure 2.

A right descent in a normalized tree is an internal node that is the right child of its parent. For $T \in \operatorname{Nor}_{n}$ we define $\operatorname{rdes}(T):=\mid$ right descents of $\left.T\right\} \mid$. A double right descent is a right descent whose parent is also a right descent. We denote by $\mathrm{NDRD}_{n}$ the set of trees in $\mathrm{Nor}_{n}$ with no double right descents.

Theorem 3. For $n \geqslant 1$ and $j \in\left\{0,1, \cdots,\left\lfloor\frac{n-1}{2}\right\rfloor\right\}$,

$$
\gamma_{j}\left(T_{n}(t)\right)=\left|\left\{T \in N D R D_{n} \mid \operatorname{rdes}(T)=j\right\}\right| .
$$

As it is illustrated in Figure 2, there are two trees in $\mathrm{NDRD}_{3}$ (for $n=3$ it happens to be equal to $\operatorname{Nor}_{3}$ ) with $\operatorname{rdes}(T)=0$ and one with $\operatorname{rdes}(T)=1$, corresponding to $\gamma_{0}=2$ and $\gamma_{1}=1$ respectively.
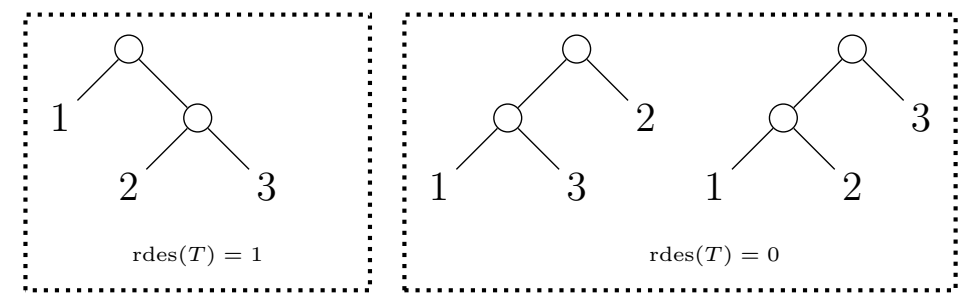

Figure 2: Set of trees in $\mathrm{NDRD}_{3}=\mathrm{Nor}_{3}$

In Section 2 we provide the proof of Theorem 3 and an additional version of Theorem 3 also in terms of normalized trees but with a statistic different than rdes. In Section 3 we provide two additional versions of Theorem 3 in terms of the Stirling permutations introduced by Gessel and Stanley in [11]. In Section 4 we discuss a generalization of the $\gamma$-positivity of $T_{n}(t)$ to the positivity of certain symmetric function in the basis of elementary symmetric functions. 


\section{Combinatorial interpretations in terms of binary trees}

Now we consider normalized trees $T$ where every internal node $x$ of $T$ has been assigned an element $\operatorname{color}(x) \in\{0,1\}$. We call an element of this set of trees a bicolored normalized tree on $[n]$. A bicolored comb is a bicolored normalized tree $T$ satisfying the following coloring restriction:

(C) If $x$ is a right descent of $T$ then $\operatorname{color}(x)=0$ and $\operatorname{color}(\mathrm{p}(x))=1$.

We denote by Comb b $_{n}$ the set of bicolored combs and by Comb ${ }_{n, i}$ the set of bicolored combs where $i$ internal nodes have been colored 1 (and $n-1-i$ colored 0 ). Figure 3 illustrates the bicolored combs on [3] grouped by the number of internal nodes that have been colored 1 .
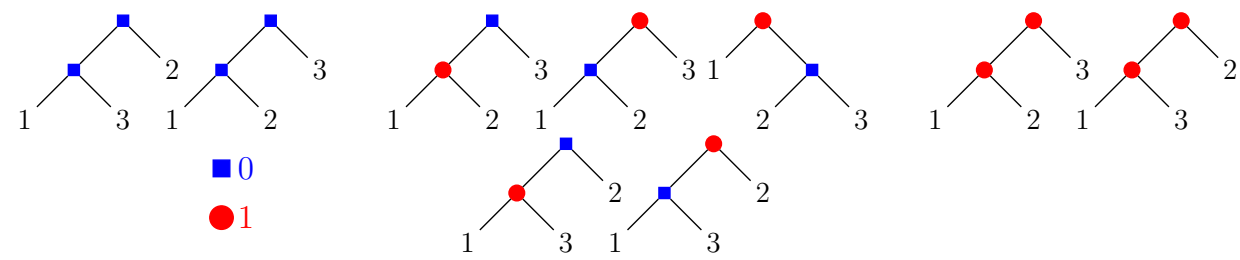

Figure 3: Set of bicolored combs on [3]

Denote by $\widetilde{T} \in \operatorname{Nor}_{n}$ the underlying uncolored normalized tree associated to a tree $T \in \mathrm{Comb}_{n}$. Note that the coloring condition (C) implies that $\widetilde{T} \in \mathrm{NDRD}_{n}$. Indeed, in a double right descent the coloring condition (C) cannot be satisfied since the parent of a double right descent is also a right descent. Also note that the monochromatic combs in $\mathrm{Comb}_{n, 0}$ and $\mathrm{Comb}_{n, n-1}$ are just the traditional left combs that are described in [21] and that index a basis for the space $\mathcal{L} i e(n)$, the multilinear component of the free Lie algebra over $\mathbb{C}$ on $n$ generators (see [21] for details). Liu [15] and Dotsenko-Khoroshkin [5] independently proved a conjecture of Feigin regarding the dimension of the multilinear component $\mathcal{L i e}_{2}(n)$ of the free Lie algebra with two compatible brackets, a generalization of $\mathcal{L} i e(n)$.

Theorem 4 ([5, 15]). For $n \geqslant 1, \operatorname{dim} \mathcal{L}_{i e_{2}}(n)=\left|\mathcal{T}_{n}\right|$.

In particular, the space $\mathcal{L} i e_{2}(n)$ has the decomposition

$$
\mathcal{L} i e_{2}(n)=\bigoplus_{i=0}^{n-1} \mathcal{L} i e_{2}(n, i),
$$

where the subspace $\mathcal{L}_{i}(n, i)$ is the component generated by certain "bracketed permutations" with exactly $i$ brackets of one of the types. Liu finds the following formula for the dimension of $\mathcal{L}_{2} e_{2}(n, i)$.

Theorem 5 ([15, Proposition 11.3]). For $n \geqslant 1$ and $i \in\{0,1, \cdots, n-1\}$,

$$
\operatorname{dim} \mathcal{L} i e_{2}(n, i)=\left|\mathcal{T}_{n, i}\right|
$$


In [1] Bershtein, Dotsenko and Khoroshkin found a basis for $\mathcal{L}_{i} e_{2}(n, i)$ indexed by the elements of $\mathrm{Comb}_{n, i}$ giving the following alternative description for the dimension of $\mathcal{L}_{i e_{2}}(n, i)$.

Theorem 6 ([1, Lemma 5.2]). For $n \geqslant 1$ and $i \in\{0,1, \cdots, n-1\}$,

$$
\operatorname{dim} \mathcal{L} i e_{2}(n, i)=\left|\operatorname{Comb}_{n, i}\right| .
$$

Corollary 7. For every $n \geqslant 1$ and $i \in\{0, \cdots, n-1\}$,

$$
\left|\operatorname{Comb}_{n, i}\right|=\left|\mathcal{T}_{n, i}\right|
$$

Problem 8. Find an explicit bijection $\operatorname{Comb}_{n, i} \rightarrow \mathcal{T}_{n, i}$, for every $n \geqslant 1$ and $i \in\{0, \cdots, n-$ $1\}$.

Theorem 9 (Theorem 3). For $n \geqslant 1$ and $j \in\left\{0,1, \cdots,\left\lfloor\frac{n-1}{2}\right\rfloor\right\}$,

$$
\gamma_{j}\left(T_{n}(t)\right)=\left|\left\{T \in N D R D_{n} \mid \operatorname{rdes}(T)=j\right\}\right| .
$$

Proof. First note that by the comments above if $T \in \mathrm{Comb}_{n}$ then its underlying uncolored tree $\widetilde{T} \in \mathrm{NDRD}_{n}$.

For a tree $T \in \mathrm{Comb}_{n}$ call free $(T)$ the number of internal nodes that are not right descents and whose right child is a leaf. Then in $\operatorname{NDRD}_{n}$ we have that

$$
\text { free }(T)+2 \operatorname{rdes}(T)=n-1 .
$$

Over the set of bicolored combs with $m$ free nodes there is a free action of $\left(\mathbb{Z}_{2}\right)^{m}$ by toggling the colors of the free nodes. Then there are $2^{m}$ bicolored combs with the same underlying tree $\widetilde{T} \in \mathrm{NDRD}_{n}$. By Corollary 7 we can write $T_{n}(t)$ as

$$
\begin{aligned}
T_{n}(t) & =\sum_{i=0}^{n-1}\left|\mathcal{T}_{n, i}\right| t^{i} \\
& =\sum_{i=0}^{n-1}\left|\operatorname{Comb}_{n, i}\right| t^{i} \\
& =\sum_{T \in \operatorname{Comb}_{n}} t^{\mid\{x \text { internal in } T \mid \operatorname{color}(x)=1\} \mid} \\
& =\sum_{\mathfrak{T} \in \operatorname{NDRD}} \sum_{T \in \operatorname{Comb}} t^{\mid\{x \text { internal in } T \mid \operatorname{color}(x)=1\} \mid} \\
& =\sum_{\mathfrak{T} \in \operatorname{NDRD}_{n}} t^{\operatorname{rdes}(\mathfrak{T})}(1+t)^{\text {free( }(\mathfrak{T})} \\
& =\sum_{\mathfrak{T} \in \operatorname{NDRD}_{n}} t^{\operatorname{rdes}(\mathfrak{T})}(1+t)^{n-1-2 \operatorname{rdes}(\mathfrak{T})} .
\end{aligned}
$$




\subsection{A second description in terms of normalized trees}

In [14] the author and Wachs studied the relation between $\mathcal{L} i e_{2}(n, i)$ and the cohomology of the maximal intervals of a poset of weighted partitions. Using poset topology techniques they found an alternative description for the dimension of $\mathcal{L} i e_{2}(n, i)$.

Define the valency $v(x)$ of a node (internal or leaf) $x$ of $T \in$ Nor $_{n}$ to be the minimal label in the subtree of $T$ rooted at $x$. For an internal node $x$ of $T$ let $L(x)$ and $R(x)$ denote the left and right children of $x$ respectively. A Lyndon node is an internal node $x$ of $T$ such that

$$
v(R(L(x)))>v(R(x))
$$

A Lyndon tree is a normalized tree in which all its internal nodes are Lyndon. We denote $\operatorname{nlyn}(T)$ the number of non-Lyndon nodes in $T$. A double non-Lyndon node is a nonLyndon node that is the left child of its parent and its parent is also a non-Lyndon node. We denote the set of trees in $\mathrm{Nor}_{n}$ with no double non-Lyndon nodes by $\mathrm{NDNL}_{n}$. A bicolored Lyndon tree is a bicolored normalized tree satisfying the coloring condition:

(L) For every non-Lyndon node $x$ of $T$ then $\operatorname{color}(x)=0$ and $\operatorname{color}(L(x))=1$.

The set of bicolored Lyndon trees is denoted $\operatorname{Lyn}_{n}$ and the set of the ones with exactly $i$ nodes with color 1 is denoted $\operatorname{Lyn}_{n, i}$.
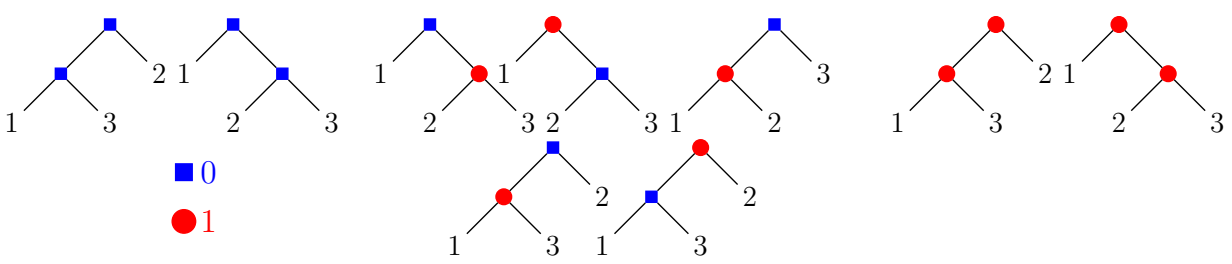

Figure 4: Set of bicolored Lyndon trees on [3]

Theorem 10 ([14, Section 5]). For every $n \geqslant 1$ and $i \in\{0, \cdots, n-1\}$,

$$
\operatorname{dim} \mathcal{L} i e_{2}(n, i)=\left|\operatorname{Lyn}_{n, i}\right| .
$$

Hence,

$$
\left|L y n_{n, i}\right|=\left|\mathcal{T}_{n, i}\right|
$$

The proof of the following theorem follows the same arguments of the proof of Theorem 3 .

Theorem 11. For $n \geqslant 1$ and $j \in\left\{0,1, \cdots,\left\lfloor\frac{n-1}{2}\right\rfloor\right\}$,

$$
\gamma_{j}\left(T_{n}(t)\right)=\left|\left\{T \in N D N L_{n} \mid \operatorname{nlyn}(T)=j\right\}\right| .
$$



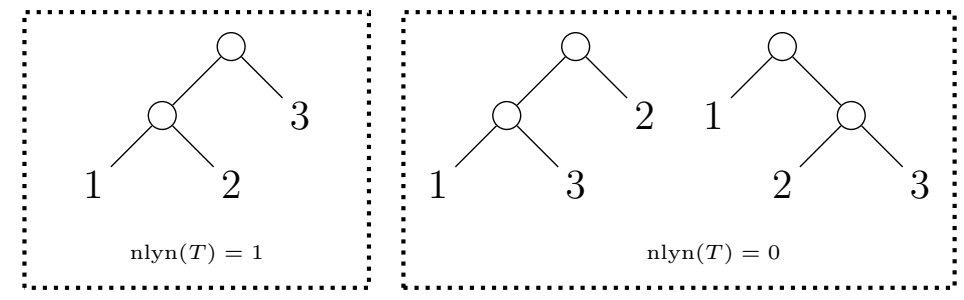

Figure 5: Set of trees in $\mathrm{NDNL}_{3}=\mathrm{Nor}_{3}$

\section{Combinatorial interpretation in terms of Stirling permuta- tions}

Consider now the set of multipermutations of the multiset $\{1,1,2,2, \cdots, n, n\}$ such that all numbers between the two occurrences of any number $m$ are larger than $m$. To this family belongs for example the permutation 12234431 but not 11322344 since 2 is less than 3 and 2 is between the two occurrences of 3 . This family (denoted $\mathcal{Q}_{n}$ ) of permutations was introduced by Gessel and Stanley in [11] and the permutations in $\mathcal{Q}_{n}$ are known as Stirling Permutations.

For a permutation $\theta=\theta_{1} \theta_{2} \ldots \theta_{2 n}$ in $\mathcal{Q}_{n}$ we say that the position $i$ contains a first occurrence of a letter if $\theta_{j} \neq \theta_{i}$ for all $j<i$, otherwise we say that it contains a second occurrence. An ascending adjacent pair in $\theta$ is a pair $(a, b)$ such that $a<b$ and in $\theta$ the second occurrence of $a$ is the immediate predecessor of the first occurrence of $b$. An ascending adjacent sequence (of length 2 ) is a sequence $a<b<c$ such that $(a, b)$ and $(b, c)$ are both ascending adjacent pairs. For example, in $\theta=13344155688776$ the ascending adjacent pairs are $(1,5),(5,6)$ and $(3,4)$ but the only ascending adjacent sequence is $1<5<6$. We denote $\mathrm{NAAS}_{n}$ the set of all Stirling permutations in $\mathcal{Q}_{n}$ that do not contain ascending adjacent sequences. Similarly, a terminally nested pair in $\theta$ is a pair $(a, b)$ such that $a<b$ and in $\theta$ the second occurrence of $a$ is the immediate successor of the second occurrence of $b$. A terminally nested sequence (of length 2) is a sequence $a<b<c$ such that $(a, b)$ and $(b, c)$ are both terminally nested pairs. For example, in $\theta=13443566518877$ the terminally nested pairs are $(1,5),(5,6)$ and $(3,4)$ but the only terminally nested sequence is $1<5<6$. We denote NTNS $_{n}$ the set of all Stirling permutations in $\mathcal{Q}_{n}$ that do not contain terminally nested sequences. For $\sigma \in \mathcal{Q}_{n}$, we denote aapair $(\sigma)$ the number of ascending adjacent pairs in $\sigma$ and tnpair $(\sigma)$ the number of terminally nested pairs in $\sigma$.

The following result in [13] relates the statistics above in $\mathcal{Q}_{n-1}$ with the ones previously discussed for $\mathrm{Nor}_{n}$.

Proposition 12 ([13, Proposition 4.8]). There is a bijection $\phi:$ Nor $_{n} \rightarrow \mathcal{Q}_{n-1}$ such that for every $T \in$ Nor $_{n}$,

1. $\operatorname{rdes}(T)=\operatorname{tnpair}(\phi(T))$

2. $\operatorname{nlyn}(T)=$ aapair $(\phi(T))$ 


$$
\begin{aligned}
& \text { 3. } \phi\left(N D R D_{n}\right)=N A A S_{n-1} \\
& \text { 4. } \phi\left(N D N L_{n}\right)=N T N S_{n-1} .
\end{aligned}
$$

Corollary 13. For $n \geqslant 1$ and $j \in\left\{0,1, \cdots,\left\lfloor\frac{n-1}{2}\right\rfloor\right\}$,

$$
\begin{aligned}
\gamma_{j}\left(T_{n}(t)\right) & =\left|\left\{T \in N T N S_{n-1} \mid \operatorname{tnpair}(T)=j\right\}\right| \\
& =\left|\left\{T \in N A A S_{n-1} \mid \operatorname{aapair}(T)=j\right\}\right| .
\end{aligned}
$$

Example 14. The Stirling permutations in $\mathcal{Q}_{2}$ are 1122, 1221 and 2211. In this particular case $\mathcal{Q}_{2}=\mathrm{NAAS}_{2}=\mathrm{NTNS}_{2}$ and the statistics in Table 2 imply that $\gamma_{0}=2$ and $\gamma_{1}=1$ are the $\gamma$-coefficients of the polynomial $T_{3}(t)$.

\begin{tabular}{|c|c|c|}
\hline $\boldsymbol{\sigma}$ & tnpair & aapair \\
\hline 1122 & 0 & 1 \\
\hline 1221 & 1 & 0 \\
\hline 2211 & 0 & 0 \\
\hline
\end{tabular}

Table 2: tnpair and aapair statistics in $\mathcal{Q}_{2}$.

\section{A comment about $\gamma$-positivity and $e$-positivity}

Let $\mathbf{x}=x_{1}, x_{2}, \ldots$ be an infinite set of variables and $\Lambda=\Lambda_{\mathbb{Q}}$ the ring of symmetric functions with rational coefficients on the variables $\mathbf{x}$, that is, the ring of power series on $\mathbf{x}$ of bounded degree that are invariant under permutation of the variables.

Define $e_{0}:=1$, for $n \geqslant 1$

$$
e_{n}:=\sum_{1 \leqslant i_{1}<i_{2}<\cdots<i_{n}} x_{i_{1}} x_{i_{2}} \cdots x_{i_{n}}
$$

and for an integer partition $\lambda=\left(\lambda_{1} \geqslant \lambda_{2} \geqslant \cdots\right)$ (i.e., a weakly decreasing finite sequence of positive integers) $e_{\lambda}:=\prod_{i} e_{\lambda_{i}}$. We call $e_{\lambda}$ the elementary symmetric function corresponding to the partition $\lambda$.

It is known that for $n \geqslant 0$, the set $\left\{e_{\lambda} \mid \lambda \vdash n\right\}$ is a basis for the $n$-th homogeneous graded component of $\Lambda$, where the grading is with respect to degree. See [16] and [19] for more information about symmetric functions.

Note that if we make the specialization $x_{i} \mapsto 0$ in $\Lambda$ for all $i \geqslant 3$ then $e_{1} \mapsto x_{1}+x_{2}$, $e_{2} \mapsto x_{1} x_{2}$ and $e_{i} \mapsto 0$ for all $i \geqslant 3$. Thus for a partition $\lambda$ of $n$ (i.e., $\sum_{i} \lambda_{i}=n$ ) the symmetric function $e_{\lambda} \mapsto 0$ unless $\lambda=\left(2^{j}, 1^{n-2 j}\right)$ for some $j \in \mathbb{N}$. In that case,

$$
e_{\left(2^{j}, 1^{n-2 j}\right)} \mapsto\left(x_{1} x_{2}\right)^{j}\left(x_{1}+x_{2}\right)^{n-2 j} .
$$

If we further replace $x_{1} \mapsto 1$ and $x_{2} \mapsto t$ we obtain

$$
e_{\left(2^{j}, 1^{n-2 j}\right)} \mapsto t^{j}(1+t)^{n-2 j}
$$


In other words, the elementary basis in two variables is equivalent to the $\gamma$ basis. A consequence of this observation is that another possible approach to conclude the $\gamma$ nonnegativity of a palindromic polynomial $f(t)$ is to find an $e$-nonnegative symmetric function $F\left(x_{1}, x_{2}, \ldots\right)$ such that $f(t)=F(1, t, 0,0, \ldots)$.

\subsection{Colored combs and comb type of a normalized tree}

A colored comb is a normalized binary tree $T$ together with a function color that assigns positive integers in $\mathbb{P}$ to the internal nodes of $T$ and that satisfies the following coloring restriction: for each internal node $x$ whose right child $R(x)$ is not a leaf,

$$
\operatorname{color}(x)>\operatorname{color}(R(x)) \text {. }
$$

Note that the set of colored combs that only use the colors 1 and 2 are the same as the bicolored combs defined in Section 2. We denote MComb $_{n}$ the set of colored combs with $n$ leaves. Figure 6 shows an example of a colored comb.

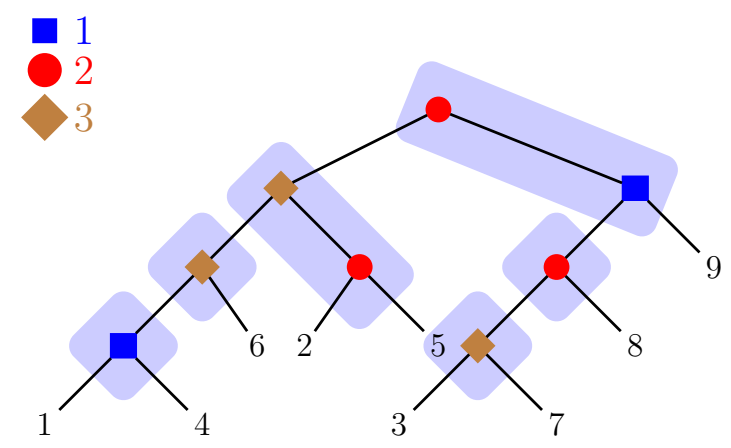

Figure 6: Example of a colored comb of comb type $(2,2,1,1,1,1)$

We can associate a type to each $\Upsilon \in$ Nor $_{n}$ in the following way: Let $\pi(\Upsilon)$ be the finest (set) partition of the set of internal nodes of $\Upsilon$ satisfying

- for every pair of internal nodes $x$ and $y$ such that $y$ is a right child of $x, x$ and $y$ belong to the same block of $\pi(\Upsilon)$.

We define the comb type $\lambda(\Upsilon)$ of $\Upsilon$ to be the integer partition whose parts are the sizes of the blocks of $\pi(\Upsilon)$.

Note that the coloring condition (4.1) is closely related to the comb type of a normalized tree. The coloring condition implies that in a colored comb $\Upsilon$ there are no repeated colors in each block $B$ of the partition $\pi(\Upsilon)$ associated to $\Upsilon$. So after choosing $|B|$ different colors for the internal nodes of $\Upsilon$ in $B$, there is a unique way to assign the colors such that $\Upsilon$ is a colored comb (the colors must decrease towards the right in each block of $\pi(\Upsilon)$ ). In Figure 6 this relation is illustrated.

For a colored comb $C$ denote $\mu(C)$ the sequence of nonnegative integers such that

$$
\mu(C)(j):=\mid\{x \text { a internal node in } C \mid \operatorname{color}(x)=j\} \mid .
$$


Let $\mathbf{x}^{\mu}:=x_{1}^{\mu(1)} x_{2}^{\mu(2)} \cdots$ and

$$
F_{\mathrm{MComb}_{n}}(\mathbf{x}):=\sum_{C \in \mathrm{MComb}_{n}} \mathbf{x}^{\mu(C)}
$$

The following theorem is a consequence of the definition of a colored comb, the definition of the symmetric functions $e_{i}(\mathbf{x})$ and the observations above (see [13]).

Theorem 15 ([13]). For $n \geqslant 1$

$$
F_{M \operatorname{Comb}_{n}}(\mathbf{x})=\sum_{T \in \text { Nor }_{n}} e_{\lambda(T)}(\mathbf{x})
$$

Note that $F_{\mathrm{MComb}_{n}}(1, t, 0,0, \ldots)=\sum_{C \in \mathrm{Comb}_{n}} t^{\mathrm{red} C}=T_{n}(t)$ and so Theorem 15 is a generalization of Theorem 3 .

Remark 16. Versions of Theorem 15 can also be given in terms of a completely different type on the set Nor $_{n}$ corresponding to a family of multicolored Lyndon trees and also in terms of colored Stirling permutations, see [13].

\section{Acknowledgments}

The author would like to thank Ira Gessel and an anonymous referee for pointing out the correct references for some of the classical results mentioned in this note.

\section{References}

[1] M. Bershtein, V. Dotsenko, and A. Khoroshkin. Quadratic algebras related to the bi-Hamiltonian operad. Int. Math. Res. Not. IMRN, (24):Art. ID rnm122, 30, 2007.

[2] P. Brändén. Sign-graded posets, unimodality of $W$-polynomials and the CharneyDavis conjecture. Electron. J. Combin., 11(2):Research Paper 9, 15 pp. (electronic), 2004/06.

[3] P. Brändén. Actions on permutations and unimodality of descent polynomials. European J. Combin., 29(2):514-531, 2008.

[4] P. Brändén. Unimodality, log-concavity, real-rootedness and beyond. ArXiv preprint arXiv:1410.6601, 2014.

[5] V. V. Dotsenko and A. S. Khoroshkin. Character formulas for the operad of a pair of compatible brackets and for the bi-Hamiltonian operad. Funktsional. Anal. $i$ Prilozhen., 41(1):1-22, 96, 2007.

[6] B. Drake. An inversion theorem for labeled trees and some limits of areas under lattice paths. ProQuest LLC, Ann Arbor, MI, 2008. Thesis (Ph.D.)-Brandeis University.

[7] Ö. Ĕ̆gecioğlu and J. B. Remmel. Bijections for Cayley trees, spanning trees, and their q-analogues. J. Combin. Theory Ser. A, 42(1):15-30, 1986. 
[8] D. Foata and M.-P. Schützenberger. Théorie géométrique des polynômes eulériens. Lecture Notes in Mathematics, Vol. 138. Springer-Verlag, Berlin-New York, 1970.

[9] D. Foata and V. Strehl. Rearrangements of the symmetric group and enumerative properties of the tangent and secant numbers. Math. Z., 137:257-264, 1974.

[10] Ś. R. Gal. Real root conjecture fails for five- and higher-dimensional spheres. Discrete Comput. Geom., 34(2):269-284, 2005.

[11] I. Gessel and R. P. Stanley. Stirling polynomials. J. Combinatorial Theory Ser. A, 24(1):24-33, 1978.

[12] I. M. Gessel and S. Seo. A refinement of Cayley's formula for trees. Electron. J. Combin., 11(2):Research Paper 27, 23, 2004/06.

[13] R. S. González D'León. On the free Lie algebra with multiple brackets. arXiv preprint arXiv:1408.5415, 2014.

[14] R. S. González D'León and M. L. Wachs. On the (co) homology of the poset of weighted partitions. arXiv preprint arXiv:1309.5527, 2013.

[15] F. Liu. Combinatorial bases for multilinear parts of free algebras with two compatible brackets. J. Algebra, 323(1):132-166, 2010.

[16] I. G. Macdonald. Symmetric functions and Hall polynomials. Oxford Mathematical Monographs. The Clarendon Press, Oxford University Press, New York, second edition, 1995.

[17] A. Postnikov, V. Reiner, and L. Williams. Faces of generalized permutohedra. Doc. Math., 13:207-273, 2008.

[18] L. W. Shapiro, W. J. Woan, and S. Getu. Runs, slides and moments. SIAM J. Algebraic Discrete Methods, 4(4):459-466, 1983.

[19] R. P. Stanley. Enumerative combinatorics. Vol. 2, volume 62 of Cambridge Studies in Advanced Mathematics. Cambridge University Press, Cambridge, 1999.

[20] R. P. Stanley. Enumerative combinatorics. Volume 1, volume 49 of Cambridge Studies in Advanced Mathematics. Cambridge University Press, Cambridge, second edition, 2012.

[21] M. L. Wachs. On the (co)homology of the partition lattice and the free Lie algebra. Discrete Math., 193(1-3):287-319, 1998. Selected papers in honor of Adriano Garsia (Taormina, 1994). 